\title{
Recombinant Human Epidermal Growth Factor Alleviates Gastric Antral Ulcer Induced by Naproxen: A Non-steroidal Anti-Inflammatory Drug
}

\author{
Ashok Raja Chairmandurai ${ }^{a}$, Srinivas Vellimedu Kanappa ${ }^{a}$, Krishna Mohan Vadrevua, \\ Uday Kumar Putchab, Vijayalakshmi Venkatesan ${ }^{\text {c, d }}$
}

\begin{abstract}
Background: To study the effect (s) of recombinant human Epidermal Growth Factor (rhEGF) on naproxen induced gastric ulcer in Wistar NIN rats.

Methods: Male Wistar NIN rats were randomly divided into six experimental groups: Group I - Control, Group II - Naproxen treated, Group III - Naproxen with rhEGF/7 days, Group IV - Naproxen without rhEGF/7 days, Group V - Naproxen with rhEGF/14 days, and Group VI - Naproxen without rhEGF/14 days. Gastric ulcer was induced with naproxen at a concentration of $80 \mathrm{mg} / \mathrm{kg}$ by oral administration. After 24 hours of induction of ulcer, rhEGF treatment was started at a concentration of $100 \mu \mathrm{g} / \mathrm{kg}$. Ulcer presence and healing were confirmed by histopathology and molecular markers.
\end{abstract}

Results: Naproxen per se induced gastric antral ulcers in Wistar NIN rats. Compared with control rats, naproxen induced rats had increased lipid peroxide content in serum. Subsequent decrease in lipid peroxide was observed in rhEGF treated groups. Treatment with rhEGF significantly resulted in healing of the ulcers, which was evident by 7 days of rhEGF treatment with total healing seen by 14 days. Significant increase in immunoreactivity for Cox-2 was observed when compared to control groups, whereas less immunoreactivity of Cox-2 was observed in rhEGF treated group. Compared with control group, naproxen induced group exhibited more

Manuscript accepted for publication May 11, 2010

${ }^{a}$ Bharat Biotech International Limited, Genome Valley, Shameerpet Mandal, Hyderabad 500 078, Andhra Pradesh, India

${ }^{b}$ Department of Pathology, National Institute of Nutrition, Jamai

Osmania, Tarnaka, Hyderabad-500 007, India

${ }^{c}$ Department of Biochemistry, Stem Cell Research Laboratory,

National Institute of Nutrition, Jamai Osmania, Tarnaka, Hyderabad-500 007, India

dCorresponding author, Email: v.venkateshan@gmail.com or v_venkateshan@hotmail.com

doi:10.4021/gr2010.05.199w gene expression of both Cox-2 and TGF beta while gene expression of Cox-2 and TGF beta in rhEGF group was comparable to control group.

Conclusions: The beneficial effects of rhEGF in the management of ulcer healing can be understood. Oral rhEGF can promote healing of the rats with gastric ulcer by stimulating Cox-2 and TGF-beta expression.

Keywords: rhEGF; Naproxen; NSAIDs; Gastric-antral ulcer; Healing; WNIN rats

\section{Introduction}

Ulcer is a debilitating disease affecting a significant proportion of the population globally [1]. Epidemiological studies show that males have about three times as many ulcers as females between the ages of fifty-five and seventy years. The etiologies of this condition are multifactorial which include Helicobacter pylori infection, NSAID toxicity, ZollingerEllison syndrome, gastrinoma, smoking and so on. Studies have shown that blood type ' $\mathrm{A}$ ' is more likely to have gastric ulcers, while those with type ' $\mathrm{O}$ ' are more likely to develop duodenal ulcers. To some extent, mental stress also plays an important role in inducing ulcers1.Ulcer healing is a complex process, which involves cell migration, proliferation, reepithelization, angiogenesis, and matrix deposition ultimately, leading to scar formation [2]. Studies in model system have helped to elucidate the pathophysiology and pathobiology underlying the gastric ulcers induced by several factors such as aspirin, alcohol, acetic acid and so on [3]. Ulcer causes inflammatory injuries in the gastric or duodenal mucosa with extension beyond the sub mucosa into the muscularis mucosa leading to imbalance between the gastro-duodenal mucosal defense mechanisms [4].

Naproxen is a non-corticosteroid drug with anti-inflammatory, antipyretic and pain relieving properties used more frequently than other NSAIDs for arthritic patients $[5,6]$. However, the major cause of concern with the long term use of naproxen is the development of the gastric antral ulcers 
Table 1. Experimental Design

\begin{tabular}{lllll}
\hline Group & $\begin{array}{l}\text { No. of } \\
\text { Animals }\end{array}$ & Treatment & Duration & Sacrifice After \\
\hline I & 6 & Distilled water & 1 day & 1 day \\
II & 6 & Naproxen & 1 day & 1 day \\
III & 6 & Group II + without rhEGF & 7 days & 7 days \\
IV & 6 & Group II + with rhEGF & 7 days & 7 days \\
V & 6 & Group II + without rhEGF & 14 days & 14 days \\
VI & 6 & Group II + with rhEGF & 14 days & 14 days \\
\hline
\end{tabular}

All the experimental conditions are as described in materials and methods. Six animals per group have been taken for this study.

$[7,8]$. New insights from basic and clinical research suggest that preservation and/or cytoprotection of gastric mucosa is of utmost importance towards the control and management of peptic ulcers. In light of these findings, it appears logical to explore for the promising molecule(s) which may serve as a beneficial tool to develop prophylactic and therapeutic remedies given the exorbitant cost of treating ulcers on the long term basis. Studies by Kim et al [9] have shown the beneficial effects of the astaxanthin - a plant derived extract which was effective in overcoming the naproxen induced gastric antral damage. Similarly, the cytoprotective effects of the growth factor such as Epidermal Growth factor (EGF) were effective towards the restoration of lesioned gastro intestinal mucosal integrity both in vivo and in vitro [10]. In line with these reports it has been demonstrated that orally administered EGF ameliorated the gastric and duodenal mucosa injury induced with the ethanol treatment [11].The Recombinant Human EGF (rhEGF) developed indigenously by Bharat Biotech International Limited has been from expression of a synthetic gene in Escherichia coli similar to the natural EGF with its purity to be $99 \%$ assessed by HPLC [12]. Further, its healing functions have been unequivocally demonstrated in diabetic foot ulcers, burns and skin grafts in the human subjects [13].

In light of these findings, it appeared logical for us to explore the beneficial effects of rhEGF against the gastric antral lesions induced with the naproxen treatment on the long term basis using biochemical, cellular and molecular approaches. Further, there are no reports to document the EGF effects on the naproxen induced gastric antral ulcers despite the fact that the gastric antrum forms the primary site of exposure. Further, understanding the healing and repair process with rhEGF also suggests its therapeutic efficacy in patients being treated with long term anti-inflammatory drugs such as NSAID.

\section{Materials and Methods}

\section{Animals}

All animal experimental procedures were approved by the Institutional Ethical Committee on animal research and the institutional guidelines were followed for the care and use of laboratory animals. Six to eight weeks male WNIN rats were obtained from the inbred colony at National Center for Laboratory Animal Sciences, Hyderabad, India. All animals were in a pathogen free environment maintained at 12 hours dark and light cycles with temperature and humidity control. The animals had free access to standard feed and water.

\section{Experimental design}

Thirty-six male WNIN rats were randomly divided into six experimental groups as given in Table 1. Naproxen treated animals received a single dose of $80 \mathrm{mg} / \mathrm{kg}$ body weight dissolved in double distilled water administered by orogastric gavage [14]. The animals were sacrificed after 24 hours (Group II), 7 days (Group III) or 14 days (Group V). The rhEGF was administered orally at the concentration of 100 $\mu \mathrm{g} / \mathrm{kg}$ body weight [14], to the 24 hours naproxen treated animals for a period of either 7 days (Group IV) or 14 days (Group VI). The Group I animals which received only double distilled water served as the controls. At the end of the experimental duration, the animals were killed by $\mathrm{CO} 2$ asphyxiation. Stomach was opened along the greater curvature, then rinsed with $0.9 \%$ saline, and the gastric antral regions were dissected out and taken for analyses.

\section{Histopathology}

The gastro-antral regions were excised from all the animals, 
Group I

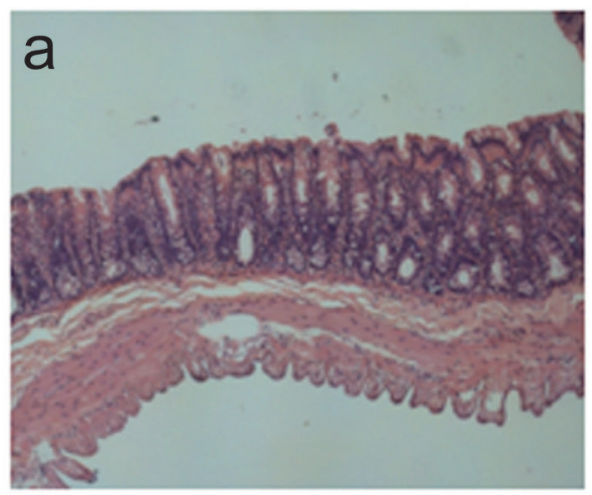

\section{Group III}

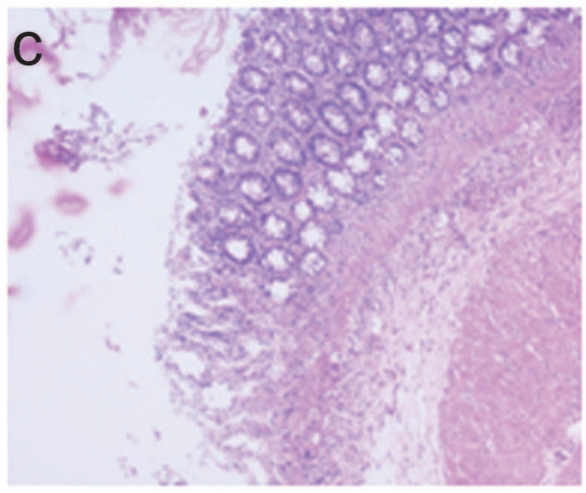

Group V

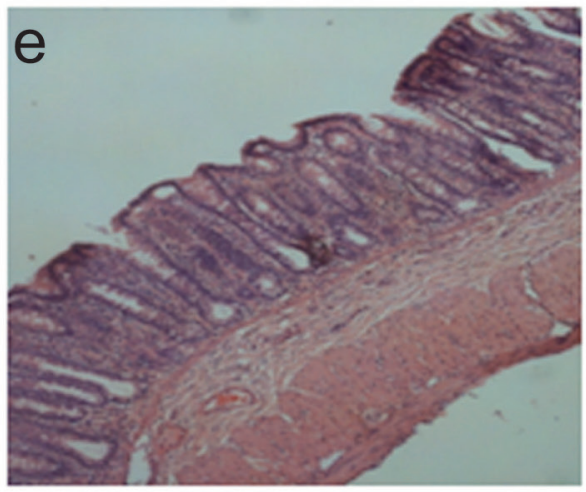

\section{Group II}

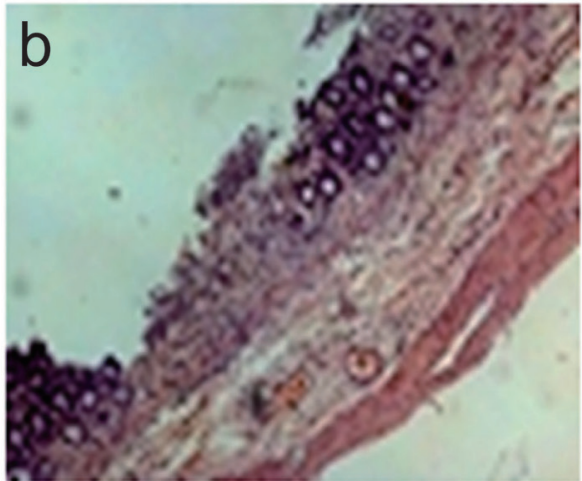

Group IV

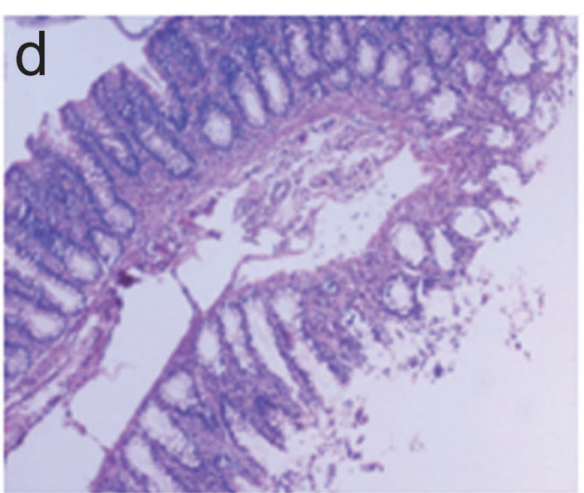

Group VI

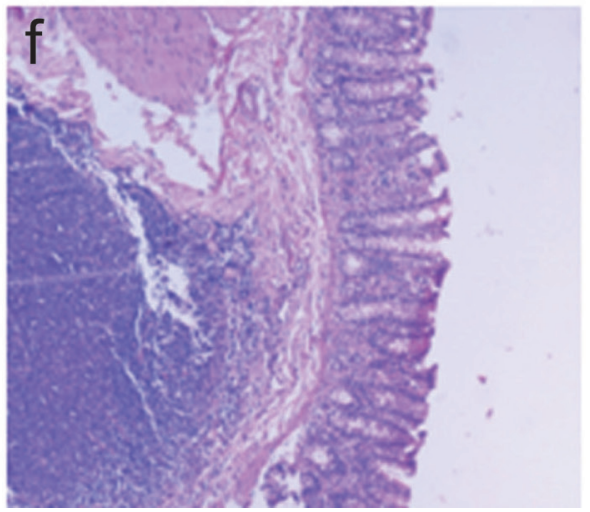

Figure 1. Histopathology. a) Normal mucosa seen in Group I animals; b) Ulceration (arrow) with inflammatory exudates and denudation of mucosal glands induced with only naproxen in Group II; c) Denudation of epithelial lining (arrow) with inflammatory cells in mucosa and submucosal layer in Group III animals (7 days); d) Mucosa of rhEGF treated animals, Group IV (7 days); e) Incomplete regeneration of mucosa (arrow) seen along with lymphoid hyperplasia in Group V animals (14 days); f) Complete regeneration of mucosa seen in the rhEGF treated animals, Group VI (14 days). Magnification is $100 \mathrm{x}$ for all the images. The data represented are from three independent identical experiments.

preserved in $10 \%$ neutral buffered formalin, and embedded in paraffin, of which $4 \mu \mathrm{m}$ thick sections were obtained using microtome (Leica RM 3040) and were subsequently stained with Hematoxylin and Eosin ( $\mathrm{H}$ and $\mathrm{E})$ by the standard 
method [15].

\section{Measurement of serum thiobarbituric acid reactive spe- cies (TBARS)}

Lipid peroxidation was estimated by measuring the thiobarbituric acid reactive species (TBARS) using 1,1,3,3-Tetramethoxypropane [16]. Briefly, 80 ul of serum from the control and experimental groups were mixed thoroughly with freshly prepared thiobarbituric acid and heated at $90^{\circ} \mathrm{C}$ for 30 minutes in a water bath, cooled to room temperature and centrifuged. The pink colored product was measured at 532 $\mathrm{nm}$ for TBARS and the MDA content expressed as nanomoles per litre of serum.

\section{Immunolocalization of Cox-2}

The paraffin sections of $4 \mu \mathrm{m}$ thickness were mounted onto poly-L-lysine coated slides and were dried overnight at $37^{\circ} \mathrm{C}$. Immunohistochemical analysis was carried out using the primary polyclonal antiserum (Goat polyclonal anti Cox2, 1:250, Santa Cruz, USA), and secondary antibody (FITC labeled Donkey anti goat - 1:500, Molecular probes, U.S.A) [17]. Images were captured on Nikon Eclipse microscope (TE 2000-S series) and the fluorescence intensity units (FIU) were corrected using appropriate controls (Primary antibody controls). The FIU have been quantitated as relative florescence units (RFU) and the experiments have been carried out independently in three sets of experiments.

\section{Semi-quantitative RT-PCR}

Total RNA was extracted from gastro-antral regions by in situ lysis of the tissues with trizol reagent. The integrity of RNA was confirmed by agarose gel electrophoresis $(1 \%)$. cDNA was prepared from 1.5 ug of total RNA using enhanced avian myeloblastosis virus reverse transcriptase (eAMV-RT) enzyme (Sigma, St. Louis, MO, USA) and the cDNA was amplified with JumpStart AccuTaq LA DNA Polymerase kit (Sigma, St. Louis, MO, USA) according to the manufacturers protocol for genes Cox-2, TGF- $\beta$ and $\beta$ actin. PCR products were separated in $1 \%$ agarose gel and stained with ethidium bromide. The band intensity was measured using the gel-documentation system (BIO-RAD U.S.A) and quantitated using 1-D analysis software (BIORAD U.S.A). Levels of mRNA expression were normalized with those of internal control $\beta$ actin.

\section{Statistical analysis}

All data have been expressed as means \pm SE. One-way analysis of variance (ANOVA) followed by posthoc LSD test with SPSS software was applied to determine the significance. P value less than 0.05 was considered statistically significant.

\section{Results}

\section{Histopathology}

The Group I animals demonstrated normal morphology with mucosal integrity of the gastro-antral region. However, the Group II animals showed gastric antral ulcers with denudation of mucosal glands and inflammatory exudates. Similarly, Group III and Group V animals showed more of denudation of epithelial lining with inflammatory cells in submucosal layer similar to that of Group II animals. However, administration of rhEGF to the naproxen treated animals ameliorated the pathological changes of gastric mucosa partially with some inflammatory changes still persisting in the gastric mucosa at 7 days (Group IV), with a total amelioration seen by 14 days (Group VI). The cytoarchitecture of the gastric antral region of the Group VI animals was comparable to Group I (Fig. 1).

\section{Measurement of serum thiobarbituric acid reactive spe- cies (TBARS)}

The TBARS levels in the serum were significantly increased in the naproxen treated animals (Group II, Group III and Group V). With rhEGF treatment, there was an appreciable decrease in the serum TBARS content which was comparable to that of the control animals. The significance was at $\mathrm{P}$ value of less than 0.05 (Fig. 2).

\section{Immunolocalization of Cox-2}

Treatment with naproxen (Group II, Group III and Group

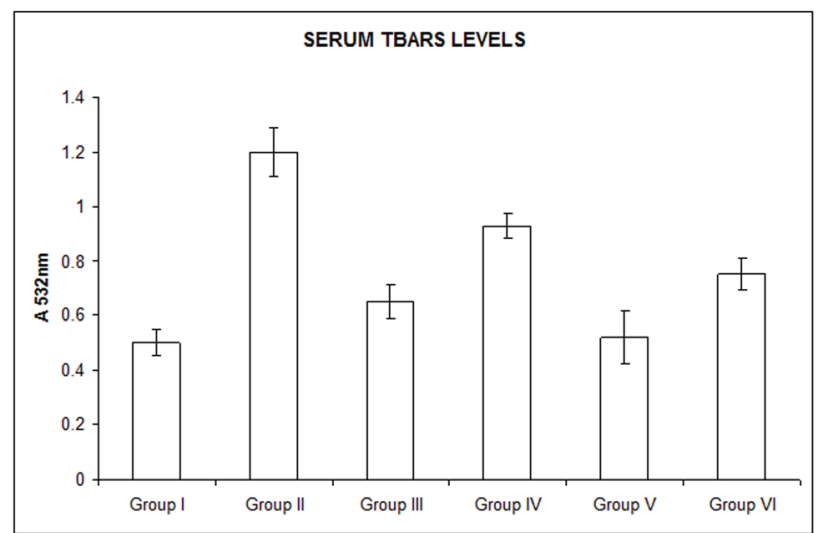

Figure 2. Measurement of serum TBARS. Serum TBARS levels were significantly increased with naproxen treatment in Group II, Group III and Group V animals. TBARS levels were decreased with rhEGF treatment in Group IV and Group VI animals which were comparable to the Group I (controls). Data are the means $\pm \mathrm{SE}$ for three independent experiments. $(\mathrm{P}<0.05$ compared to Group I, Group IV and Group VI by one way ANOVA). 

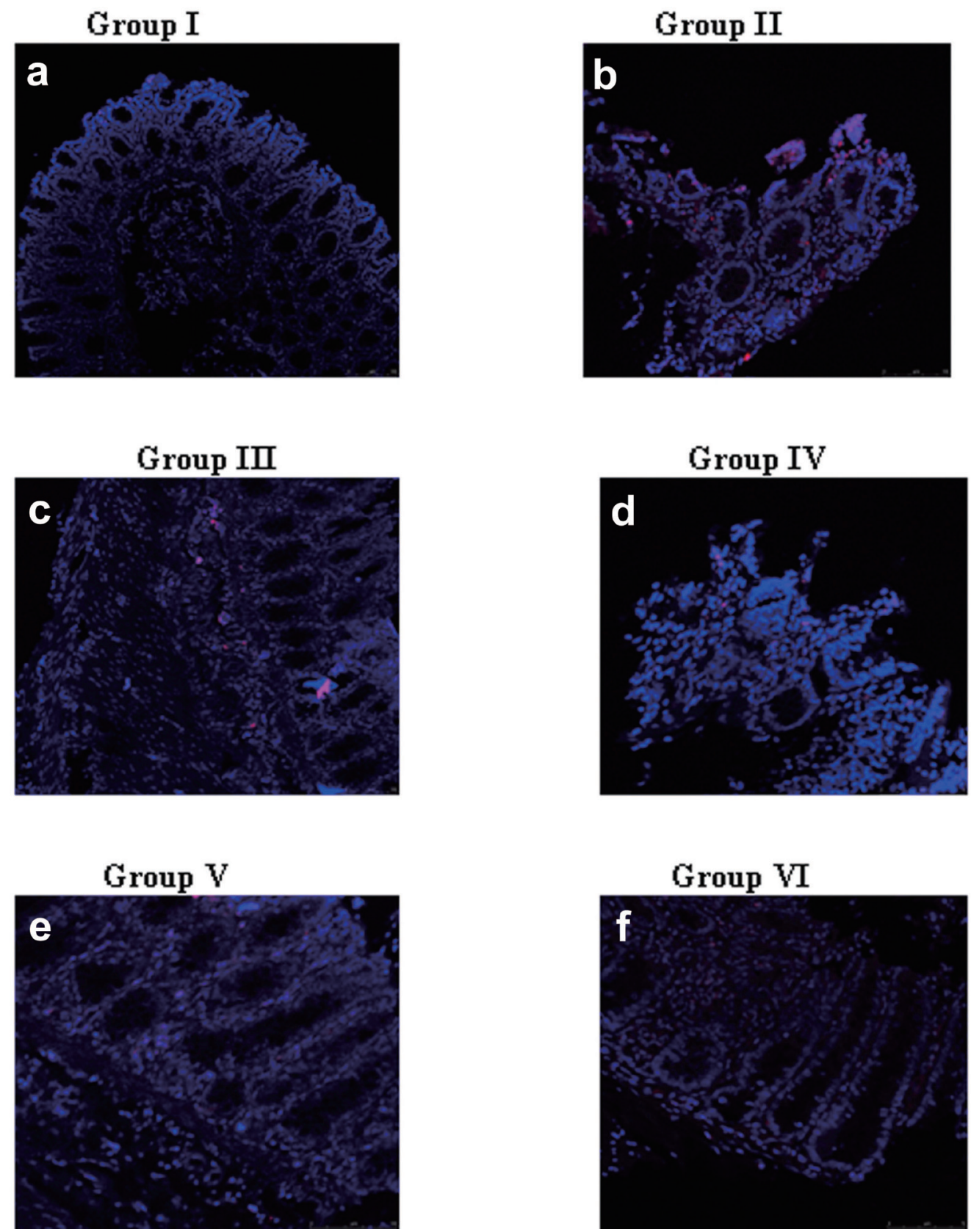

Figure 3. Immunolocalization of Cox-2. Cox-2 localization was weak in: a) Group I animals; d) rhEGF treated Group IV (7days); f) rhEGF treated Group VI (14days). However, a predominant localization was seen in naproxen treated animals such as: b) Group II; c) Group III; e) Group V. The data represented are from three independent identical experiments and the images have been captured with Nikon Inverted fluorescence microscope TE 2000-S series.

V) demonstrated Cox-2 localization seen predominantly in the ulcerated region of the gastric antral tissue. Interestingly, with rhEGF treatment, the Cox-2 localization was reduced to a larger extent (Group IV) and was almost absent and comparable to that of the controls by 14 days of treatment (Group VI) (Fig. 3). The relative fluorescent unit (RFU) of the Cox-2 immunolocalization in control and the experimental groups was calculated (data not shown).

\section{Semi-quantitative RT-PCR}

Treatment with naproxen (Group II, Group III and Group $\mathrm{V})$ showed an upregulation in the expression of Cox-2 and TGF- $\beta$. Administration of rhEGF to the naproxen treated 
a

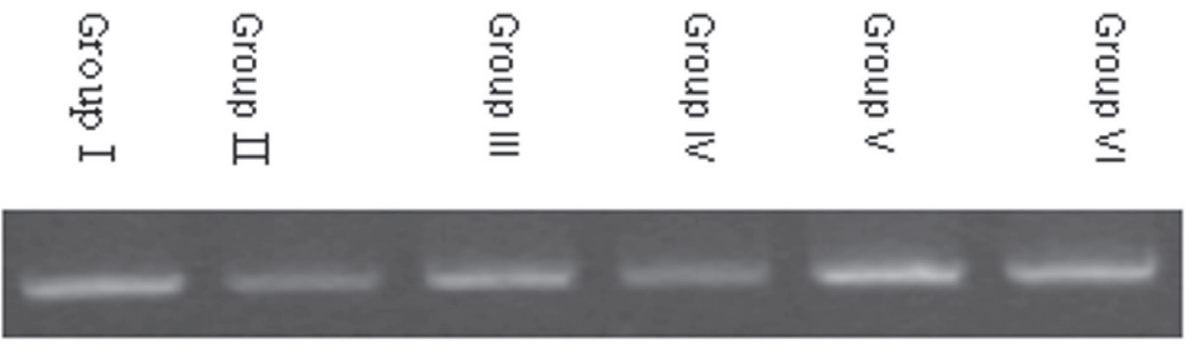

Cox-2

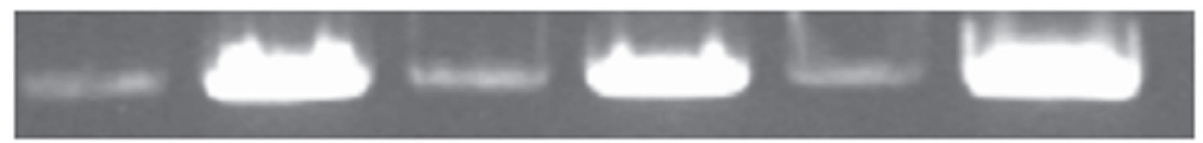

TGF-beta

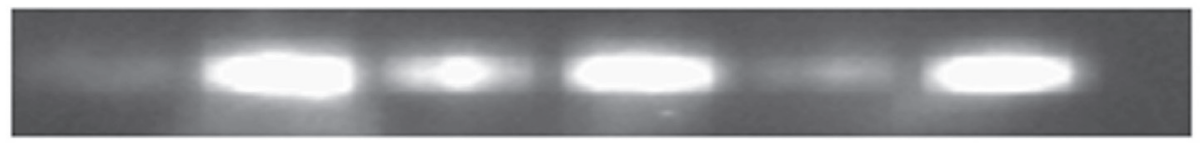

b
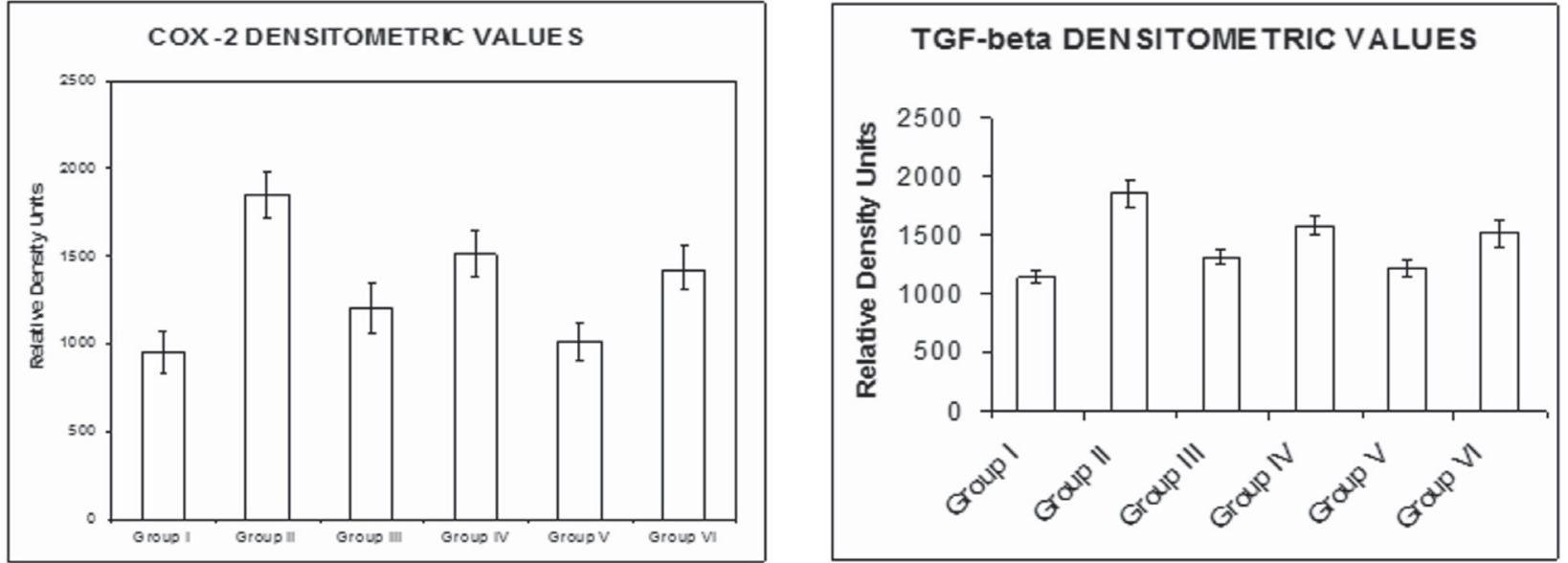

Figure 4. Semi-quantitative RT-PCR. a) An increase in the expression of both Cox-2 and TGF- $\beta$ were seen with naproxen treatment in Group II, Group III and Group V animals. Administration of rhEGF decreased the expression of Cox-2 and TGF- $\beta$ in Group IV and Group VI animals which were similar to the Group I (controls); b) The densitometry analysis of the RT-PCR data for Cox-2 and TGF- $\beta$. Data are the means \pm SE for three independent experiments. $(P<0.05$ compared to Group I, Group IV and Group VI by one way ANOVA).

animals decreased the expression of both the genes Cox-2 and TGF- $\beta$ which was more significant in Group VI animals similar to the Group I animals. However, the reduction in the expression of the Cox- 2 and TGF- $\beta$ was of lesser magnitude in the 7 days of rhEGF treatment (Group IV). $\beta$ actin was used as the house-keeping gene in each assay (Fig. 4a). The quantitative changes in the expression for both Cox-2 and TGF- $\beta$ have been indicated by densitometric scanning (Fig. $4 b)$.

\section{Discussion}

The present study has been carried out to explore the heal- ing effects of rhEGF in the naproxen induced gastric antral ulcers treated either for 7 days or for 14 days. Earlier studies have reported the efficacy of EGF in negating the detrimental effects of the gastric mucosal ulcers both in acute and chronic conditions treated with ethanol [11]. However, these studies are limited at several levels in understanding the cytoprotection and repair mechanism(s) underlying the healing process [18]. The rhEGF which has been developed indigenously by BBIL India [12] have proven to be effective in the healing of diabetic foot ulcers, skin burns and wound healing when tested in the human subjects [13]. Literature survey shows that there have been no studies undertaken to implicate the beneficial effects of rhEGF in the management of gastric antral ulcers induced with NSAID keeping in view 
of the fact that NSAIDs have been frequently used as antiinflammatory drugs for arthritic patients and that gastric ulceration is widely prevalent especially in the antral region [9]. Our present study forms a novel approach to investigate the pathology, biochemical, cellular and molecular changes associated in the naproxen induced gastric antral ulcers and their healing with rhEGF offering a suitable model system to the human situation.

EGF is mitogenic and its proliferative effect is believed to contribute to the maintenance of mucosal integrity within the gastrointestinal tract [18]. In our present study, the lag time from ulcer induction to complete gastric healing with rhEGF treatment was 14 days and the benefits of such agents would include a higher therapeutic index and lower toxicity than conventional therapies [13]. Further, exogenous EGF administration given parenterally or orally, stimulated the growth of gastric/duodenal mucosa and was shown to be effective in restoration of the damage induced with acetic acid/ ethanol treatment [19]. However, there are other reports to show that oral administration of EGF at 30 and $100 \mu \mathrm{g} / \mathrm{kg}$ bodyweight for 2 weeks had no effect on the ulcer healing in Donryu rats with gastric ulcers induced by submucosal injection of acetic acid into the antral region. These variations probably could be attributed to the different strains of animals, method of ulcer induction, severity of ulcer formation used and duration of ulcer treatment [20].

We examined the histopathology of the gastric antral tissues by Hematoxylin and Eosin of the control and the experimental Groups. The results undoubtedly demonstrated the healing rendered by rhEGF as evidenced by the normalization of cytoarchitecture of the gastric mucosa by 14 days with some inflammatory changes still persisting in the early phase of the treatment (7 days) (Fig. 1). Studies have shown that the ulcerative lesions of gastro-antral tract are one of the major side effects of NSAID(s) and limit their usage on longterm basis [9]. Supporting these studies, our data also substantiate that with naproxen treatment, gastric antral lesions underlying the ulcerative changes are appreciable. This has been manifested with denudation of mucosal glands and inflammatory exudates seen in Group II, Group III and Group $\mathrm{V}$ animals. Naproxen is a non-corticosteroid drug with antiinflammatory and antipyretic effects causing erosions, antral ulceration and petechial bleeding in the mucosa of stomach $[5,6]$. It has been demonstrated that the production of oxygen free radicals and lipid peroxidation plays a crucial role in the development of the gastric antral ulcers induced by NSAIDs [7, 8]. In similar lines, we have also demonstrated an increase in the lipid peroxides when treated with naproxen (NSAIDs), which was negated by the beneficial effects of oral administration of rhEGF. Further, the beneficial effects to overcome the naproxen induced rise in lipid peroxide seen specifically in gastric antral ulcers with astaxanthin treatment are of therapeutic efficacy [9].

The role of Cox-2 in ulcer and healing process has been well documented [21]. However, there are conflicting reports in literature attributed to the differences in the specificity of the antibodies used or the species variations [22, 23], where the Cox-2 expression has been demonstrated even in the normal gastric mucosa. However, our present data are in line with the studies reported by Lajoie et al [24] which correlates for the increased Cox-2 localization attributed to the mucosal lesions seen in the gastric antral ulcers induced with naproxen [25-27]. Interestingly, with the total amelioration of the gastric mucosa seen by 14 days (Group VI), vis-avis, the Cox-2 localization was minimal or non detectable and comparable to the normal gastric mucosa of the controls. This suggests the process of healing and repair induced with rhEGF treatment.

Ulcer healing is a complex process which involves cascade of events and interactions regulated by the expression of several factors. Studies by Satoru [28] have reported an increase in the expression of TGF- $\beta$ in tissue repair and remodeling process akin to our present data demonstrating the increased TGF- $\beta$ and Cox- 2 expression in naproxen induced gastric lesions. The healing of the gastric antral ulcers which was almost evident by 14 days of rhEGF treatment correlated well with the decreased TGF- $\beta$ and Cox- 2 mRNA expression. Parallelly, Tominga et al [29] have also correlated the TGF- $\beta$ mRNA expression to the wound and healing process. In addition, there are some recent studies showing an induction in the HGF expression synergistic with Cox-2 expression in the gastric ulcer healing process [30, 31]. Further, the use of specific inhibitors of Cox-1 and Cox-2 such as NS-398 and indomethacin have shown to impair the healing of ulcer which suggests for an important role of endogenous growth factors in mucosal repair [4].

In conclusion, the present study forms the basis to be reported for the first time the efficacy of rhEGF in overcoming/ mitigating the gastro-antral ulcer induced with naproxen. In this experimental model, we have attempted to correlate the ulcer healing process by approaches such as histopathology, lipid peroxide levels, Cox-2 immunolocalization and expression of the tissue healing markers such as Cox- 2 and TGF- $\beta$ genes. We believe that exploring the potential of rhEGF to understand the mechanism(s) (biochemical, cellular and molecular) of healing carried out in the model system will help in advancing our knowledge towards the management of antral gastric ulcers.

\section{Acknowledgments}

We thank Director National Institute of Nutrition (NIN) for permitting the collaborative study between NIN and BBIL Hyderabad, India, and for the intra mural grant support. We thank Dr. N.V Giridharan, Director Grade Scientist, NCLAS, for extending the support to carry out animal experiments. Our thanks also give to Chairman and Managing Director of 
BBIL for the financial support and for providing the rhEGF for the work. We thank Dr. M. Raghunath for the valuable corrections in manuscript. We acknowledge inputs given by Mr. Santhosh Tiwari, Owaisi Hospital and Research Center, Hyderabad, and Mr. Rajkiran Reddy B, Himadri Singh, for the technical help in the manuscript processing.

\section{Grant Support}

Institutional

\section{References}

1. Holtmann G, Howden CW. Review article: management of peptic ulcer bleeding-the roles of proton pump inhibitors and Helicobacter pylori eradication. Aliment Pharmacol Ther 2004;19 Suppl 1(66-70).

2. Jones MK, Tomikawa M, Mohajer B, Tarnawski AS. Gastrointestinal mucosal regeneration: role of growth factors. Front Biosci 1999;4(D303-309.

3. Lee A. Animal models of gastroduodenal ulcer disease. Baillieres Best Pract Res Clin Gastroenterol 2000;14(1):75-96.

4. Konturek SJ, Konturek PC, Brzozowski T. Prostaglandins and ulcer healing. J Physiol Pharmacol 2005;56 Suppl 5(5-31).

5. Suwa T, Urano H, Kohno Y, Suzuki A, Amano T. Comparative studies on the gastrointestinal lesions caused by several nonsteroidal anti-inflammatory agents in rats. Agents Actions 1987;21(1-2):167-172.

6. Calhoun W, Gilman SC, Datko LJ, Copenhaver TW, Carlson RP. Interaction studies of tilomisole, aspirin, and naproxen in acute and chronic inflammation with assessment of gastrointestinal irritancy in the rat. Agents Actions 1992;36(1-2):99-106.

7. Parks DA. Oxygen radicals: mediators of gastrointestinal pathophysiology. Gut 1989;30(3):293-298.

8. Yoshikawa T, Naito Y, Ueda S, Oyamada H, Takemura T, Yoshida N, Sugino S, et al. Role of oxygen-derived free radicals in the pathogenesis of gastric mucosal lesions in rats. J Clin Gastroenterol 1990;12 Suppl 1(S6571.

9. Kim JH, Kim YS, Song GG, Park JJ, Chang HI. Protective effect of astaxanthin on naproxen-induced gastric antral ulceration in rats. Eur J Pharmacol 2005;514(1):5359.

10. Marti U, Burwen SJ, Jones AL. Biological effects of epidermal growth factor, with emphasis on the gastrointestinal tract and liver: an update. Hepatology 1989;9(1):126-138.

11. Xu CD, Gan RB, Chen SN, Jiang SH, Xu JY. Protection of gastric mucosa from ethanol induced injury by recombinant epidermal growth factor in rats. World $\mathrm{J}$ Gastroenterol 1998;4(5):437-438.

12. Sharma K, Babu PV, Sasidhar P, Srinivas VK, Mohan VK, Krishna E. Recombinant human epidermal growth factor inclusion body solubilization and refolding at large scale using expanded-bed adsorption chromatography from Escherichia coli. Protein Expr Purif 2008;60(1):714.

13. Vijay V, Sharad P, Sekar N, Murthy G.S.R. A Phase III study to evaluate the safety and efficacy of Recombinant Human Epidermal Growth Factor (REGEN- D150) in healing of Diabetic foot ulcers. Wounds 2006; 18(7): 186-196.

14. Susan N.E, Andre B, Webb M, Mark J.S.M, John LW (1998) Bacteria rapidly colonize and modulate healing of gastric ulcers in rats. Am J Physiology- Gastrointest Liver Physiol 1998; 275 (3): 425-432.

15. Raghuramulu N, Madhavan Nair K, Kalyanasundaram S. Histopathology: A manual of laboratory techniques, 2nd edition. National Institute of Nutrition, India: NIN press; 2003. pp 298-299.

16. Jiang P, Chang L, Pan CS, Qi YF, Tang CS. Protective role of metallothionein in stress-induced gastric ulcer in rats. World J Gastroenterol 2005;11(18):2739-2743.

17. Dorisetty RK, Kiran SG, Umrani MR, Boindala S, Bhonde RR, Venkatesan V. Immuolocalization of nestin in pancreatic tissue of mice at different ages. World $\mathrm{J}$ Gastroenterol 2008;14(46):7112-7116.

18. Miller CA, Debas HT. Epidermal growth factor stimulates the restitution of rat gastric mucosa in vitro. Exp Physiol 1995;80(6):1009-1018.

19. Konturek SJ, Dembinski A, Warzecha Z, Brzozowski T, Gregory H. Role of epidermal growth factor in healing of chronic gastroduodenal ulcers in rats. Gastroenterology 1988;94(6):1300-1307.

20. Kuwahara Y, Sunagawa Y, Imoto Y, Okabe S. Effects of orally administered human epidermal growth factor on natural and delayed healing of acetic acid-induced gastric ulcers in rats. Jpn J Pharmacol 1990;52(1):164-166.

21. Iseki S. Immunocytochemical localization of cyclooxygenase- 1 and cyclooxygenase- 2 in the rat stomach. Histochem J 1995;27(4):323-328.

22. Mayoral R, Molla B, Flores JM, Bosca L, Casado M, Martin-Sanz P. Constitutive expression of cyclo-oxygenase 2 transgene in hepatocytes protects against liver injury. Biochem J 2008;416(3):337-346.

23. Jackson LM, Wu KC, Mahida YR, Jenkins D, Hawkey CJ. Cyclooxygenase (COX) 1 and 2 in normal, inflamed, and ulcerated human gastric mucosa. Gut 2000;47(6):762-770.

24. Lajoie S, Sirois J, Dore M. Induction of cyclo-oxygenase-2 expression in naturally occurring gastric ulcers. J Histochem Cytochem 2002;50(7):923-934.

25. Mizuno H, Sakamoto C, Matsuda K, Wada K, Uchida T, 
Noguchi H, Akamatsu T, et al. Induction of cyclooxygenase 2 in gastric mucosal lesions and its inhibition by the specific antagonist delays healing in mice. Gastroenterology 1997;112(2):387-397.

26. Shigeta J, Takahashi S, Okabe S. Role of cyclooxygenase-2 in the healing of gastric ulcers in rats. J Pharmacol Exp Ther 1998;286(3):1383-1390.

27. Takahashi S, Shigeta J, Inoue H, Tanabe T, Okabe S. Localization of cyclooxygenase- 2 and regulation of its mRNA expression in gastric ulcers in rats. Am J Physiol 1998;275(5 Pt 1):G1137-1145.

28. Takahashi S, Kobayashi N, Okabe S. Regulation by endogenous interleukin-1 of mRNA expression of healingrelated factors in gastric ulcers in rats. J Pharmacol Exp
Ther 1999;291(2):634-641.

29. Tominaga K, Arakawa T, Kim S, Iwao H, Kobayashi $\mathrm{K}$. Increased expression of transforming growth factorbetal during gastric ulcer healing in rats. Dig Dis Sci 1997;42(3):616-625.

30. Kinoshita Y, Nakata H, Hassan S, Asahara M, Kawanami C, Matsushima Y, Naribayashi-Inomoto Y, et al. Gene expression of keratinocyte and hepatocyte growth factors during the healing of rat gastric mucosal lesions. Gastroenterology 1995;109(4):1068-1077.

31. Takahashi M, Ota S, Hata Y, Mikami Y, Azuma N, Nakamura T, Terano A, et al. Hepatocyte growth factor as a key to modulate anti-ulcer action of prostaglandins in stomach. J Clin Invest 1996;98(11):2604-2611. 